\title{
Emergence of Gamma distributions in granular materials and packing models
}

\author{
T. Aste and T. Di Matteo \\ Department of Applied Mathematics, The Australian National University, 0200 Canberra, ACT, Australia
}

(Received 19 June 2007; revised manuscript received 3 December 2007; published 29 February 2008)

\begin{abstract}
We study the distribution of volume fluctuations in experiments and numerical simulations concerning equal-sized sphere packings prepared with different techniques. We show that the distribution of the local volumes (Voronoï cells) and also the distributions of the global volumes (whole samples) follow remarkably well a shifted and rescaled Gamma distribution that we name a $k$-Gamma distribution. Such agreement is robust over a broad range of packing fractions and it is observed for several distinct systems. This distribution is characterized by the average packing fraction and a shape parameter " $k$ " which is very sensitive to changes in the structural organization. A statistical mechanics approach predicts such $k$-Gamma distribution at statistical equilibrium and it links the parameter $k$ with the number of elementary cells which are exchanging volume during the system preparation. The thermodynamical equivalent of $k$ and its relation with the "granular temperature" are also discussed.
\end{abstract}

DOI: 10.1103/PhysRevE.77.021309

PACS number(s): 45.70. $-\mathrm{n}, 45.70 . \mathrm{Cc}, 81.05 . \mathrm{Rm}$

\section{INTRODUCTION}

Granular materials are central in a very wide range of domains from agriculture to the pharmaceutical industry, but, despite such a relevance in most fields of human activity and their ubiquitous presence in scientific research areas, their behavior and properties remain elusive. Granular materials differ from all the other materials: they can flow like liquids under some conditions and act as solids under others [1-3]. Their intrinsic complexity is a consequence of the fact that they are composed of many pieces (the grains) that can assemble in large structures behaving collectively. The study of granular materials has forced scientists to rethink established classifications of matter and to reformulate statistical mechanics in a new context [4-26]. These studies are springing out new ideas that are advancing the understanding of large classes of complex materials from composites to foams.

It is well known since ancient times that different preparation methods can produce granular packings with different packing fractions. Mechanically stable packing of equal sized spheres can be prepared in a range of packing fractions within these two limits: $\rho_{R L P} \sim 0.555$ and $\rho_{R C P} \sim 0.645$, which are respectively known as random loose packing limit and random close packing limit. Reproducible packing fractions in the whole range between the two limits can be, for instance, prepared by means of a fluidized bed technique using different flow rates $[20,27,28]$. In this paper, we report experimental data from six samples (A-F) composed of acrylic beads prepared in air with packing fractions between $\rho \sim 0.586$ and $\rho \sim 0.640$ [29-31]. We also report data from 12 samples made of glass beads in water, prepared at packing fractions between 0.56 and 0.60 by means of a fluidized bed technique. Furthermore, we investigate numerically systems of Newtonian hard spheres which are compressed within a box that is shrinking in size at different rates [32-34] obtaining reproducible packing fractions in the whole range below $\rho \sim 0.66$. In general, for any given preparation protocol, one generates configurations with reproducible packing fractions that vary in a narrow range around an average characteristic value.
In the present study we analyze the fluctuations of such reproducible packing fractions within the framework of a statistical mechanics approach. Indeed, from a statistical mechanics perspective such fluctuations are a measure of the way in which the system is exploring the accessible phasespace. We predict that at statistical equilibrium the volume fluctuations must follow a Gamma distribution [35] in the variable $V-V_{\min }$, where $V_{\min }$ is the minimum attainable volume. This distribution has a shape parameter $k$ and a scale parameter $\left(\bar{V}-V_{\min }\right) / k$ with $\bar{V}$ being the average volume. We name such a function $k$-Gamma distribution. We find that despite the great diversity in the systems studied all the experiments and simulations follow extremely well the predicted $k$-Gamma behavior. This remarkable agreement is observed both at the local level of a single grain and at the global level of the whole sample. The most relevant result is the discovery that the parameter $k$ is very sensitive to the system's properties and preparation. In particular, we observe sharp variations of $k$ at the $\rho_{R L P}$ and $\rho_{R C P}$ limiting densities.

The paper is organized as follows. The system entropy is introduced in Sec. II and a maximum-entropy prediction for the probability of a given state is derived. The probability density function for the system's volume fluctuations is explicitly calculated in Sec. III. In Sec. IV, the concept of "granular temperature" and the links with volume fluctuations at equilibrium are discussed. In Sec. V, it is shown that the theoretical prediction for the probability density function for local volumes at the grain levels (Voronoï cells) reproduces extremely well both experimental and simulation data. Moreover, we show that the theory predicts also the global fluctuations on the scale of the whole system. The signature of transitions occurring at the random loose packing limit and at the random close packing limit are highlighted by studying the changes occurring at these densities in the quantity $k$ which is shown to be analogous to the specific heat for these systems. Conclusions and perspectives are given in Sec. VI. 


\section{EQUILIBRIUM PREDICTION FOR THE STATE PROBABILITIES}

In analogy with the Edwards' original approach [4-6,13,36,37] we consider a granular system which is prepared at a given packing fraction by means of a specific preparation protocol. We consider only the configurations when the grains are at rest in their final positions. We study the ensemble of systems at statistical equilibrium where the statistical properties are stationary and the volume occupied in each system is fluctuating around an average characteristic value $\bar{V}$ which is associated with the kind of preparation method. We adopt a "coarse grained" description of the system state in terms of the total occupied volume $V$. [Note that, for $N$ equal spheres with unitary diameters, the packing faction is simply related to the volume by $\rho=\pi N /(6 V)]$. In this approach, the volume plays the role of the energy in conventional statistical mechanics. Analogously with conventional statistical mechanics we consider the canonical ensemble and the Gibbs entropy:

$$
S=-\sum_{V} p(V) \ln p(V)+\sum_{V} p(V) S(V) .
$$

Here $p(V)$ is the probability of a state with volume $V$ and $S(V)$ is the entropy of the state $V$. When all the microscopic states are a priori equiprobable, the expression for $S(V)$ is

$$
S(V)=\ln \Omega(V),
$$

with $\Omega(V)$ the number of microscopic states which are classifiable under the same coarse grained state $V$.

We can calculate $p(V)$ by searching for the functional form of the probability distribution function which maximizes the entropy. Such maximization must be done under the condition that the average occupied volume is equal to $\bar{V}$. This yields

$$
p(V)=\frac{\Omega(V) e^{-V / \chi}}{\sum_{V^{\prime}} \Omega\left(V^{\prime}\right) e^{-V^{\prime} / \chi}},
$$

with $\chi^{-1}$ a Lagrange multiplier fixed by the constraint on the average volume:

$$
\bar{V}=\langle V\rangle=\sum_{V} V p(V)
$$

Equation (3) is the-almost unavoidable-outcome of any classical equilibrium statistical mechanics theory. The challenge is now to compute the number of microscopic states $\Omega(V)$ associated with coarse-grained states which occupy a total volume $V$. To this end we must introduce some further assumptions as discussed in the next section.

\section{SPACE PARTITIONS}

Let us image to subdivide the packing into a set of $k$ local "elementary cells." Such cells can be viewed as the portions of the physical space associated to local configurations in the granular pack. Let us call $\mathbf{c}$ the properties of such cells, and let us assume that the system's microscopic state can be clas- sified in terms of these properties: $\Psi=\left\{\mathbf{c}_{1}, \mathbf{c}_{2}, \ldots, \mathbf{c}_{k}\right\}$. The phase-space volume $\Omega(V)$ is associated with all the microscopic configurations $\Psi$ which occupy a total volume $V$. If we introduce the further assumptions that the cells can have arbitrary volumes larger than $v_{\text {min }}$ under the condition that the whole system must occupy a total volume $V$ and if we assume that the cell properties $\mathbf{c}_{i}$ are either completely determined by their volumes $v_{i}$ or they are independent from $v_{i}$, then $\Omega(V)$ can be computed exactly:

$$
\begin{aligned}
\Omega(V)= & \frac{1}{\Lambda^{3 k}} \int_{v_{\min }}^{V} d v_{1} \int_{v_{\min }}^{V} d v_{2} \cdots \int_{v_{\min }}^{V} d v_{k} \delta \\
& \times\left(v_{1}+v_{2}+\cdots+v_{k}-V\right)=\frac{\left(V-k v_{\min }\right)^{k-1}}{\Lambda^{3 k}(k-1) !},
\end{aligned}
$$

with $\Lambda$ a constant analogous to the Debye length. Substituting into Eq. (3) and by using Eq. (4) we obtain $\chi=(\bar{V}$ $\left.-k v_{\min }\right) / k$ and the probability

$$
p(V)=f(V, k)=\frac{k^{k}}{\Gamma(k)} \frac{\left(V-V_{\min }\right)^{(k-1)}}{\left(\bar{V}-V_{\min }\right)^{k}} \exp \left(-k \frac{V-V_{\min }}{\bar{V}-V_{\min }}\right),
$$

with $V_{\min }=k v_{\min }$. The function $f(V, k)$ is the probability density function to find a packing of $k$ elementary cells occupying a volume $V$, in a set of experiments where the average occupied volume is $\bar{V}$. Note that Eq. (6) is valid for any $k$ and it holds even in the limit $k=1$. Indeed, the observable system can be any arbitrary subset of a larger system. Moreover, the experiment can be performed either on several different independent systems or-equivalently-on several noninteracting subsets of a large system. Equation (6) is a Gamma distribution in the variable $V-V_{\min }$; it is characterized by a shape parameter $k$ and a scale parameter $\left(\bar{V}-V_{\min }\right) / k[35]$. In the following we will refer to such a function as $k$-Gamma distribution. In the literature empirical fits with Gamma distributions have been previously reported for two-dimensional Voronoï networks [38], and for Voronoï partitions from random Poisson points in three dimensions [39]. Interestingly, the same distribution has been observed in a statistical study of crumpled paper [40].

The average volume from the distribution $f(V, k)$ coincides with $\bar{V}$ and the variance is

$$
\sigma_{v}^{2}=\frac{\left(\bar{V}-V_{\min }\right)^{2}}{k}
$$

which is a useful relation to empirically evaluate $k$ from a set of volume measurements: $k=\left(\bar{V}-V_{\min }\right)^{2} / \sigma_{v}^{2}$. We will make use of this relation in Sec. V.

By substituting Eqs. (5) and (6) into Eq. (1), we can also derive the following explicit expression for the entropy:

$$
S=k\left[1+\ln \left(\frac{\bar{V}-V_{\min }}{k \Lambda^{3}}\right)\right] \text {. }
$$




\section{THE “DEGREES” OF SPACE PARTITION}

Following Edwards' ideas [4,36], a granular temperature can be inferred from an analogy with the thermodynamical relation $\beta=1 /\left(k_{B} T\right)=\partial$ (entropy) $/ \partial$ (energy) $[4,36]$, by substituting the volume to the role played by the energy in thermodynamical systems. In the formalism of this paper this can be written as $\beta_{g r}=\chi^{-1}=\partial S / \partial \bar{V}$. From Eqs. (1) and (5) we have

$$
\beta_{g r}^{-1}=\chi=\frac{\bar{V}-V_{\min }}{k} ;
$$

therefore the Edwards' compactivity $\chi[4,36]$ is the average free volume per elementary cell, implying that the granular temperature is a measure of the kind and the degree of space partition into elementary cells. For a given system at a given packing fraction, the granular temperature is univocally associated to the quantity $k$. By using Eqs. (7) and (9) we have

$$
\chi=\frac{\sigma_{v}^{2}}{\bar{V}-V_{\min }} .
$$

The present approach provides therefore a very effective and practical way to compute Edward's compactivity from measures of volume fluctuations.

In terms of the compactivity $\chi$ the expression for the cell volume's distribution [Eq. (6)] can be written in the more compact form:

$$
p(V)=\frac{\Omega(V)}{\Omega(\chi)} e^{-V / \chi},
$$

which recalls the equivalent expression in classical thermodynamics, with $\Omega(V)$ given by Eq. (5) and $\Omega(\chi)$ $=\Omega_{0} \chi^{k} \exp \left(-V_{\min } / \chi\right)$.

The volume fluctuations can be directly calculated from the $k$-Gamma distribution in Eq. (6) and one can verify that the correct relation between compactivity and fluctuations is attained:

$$
\chi^{2} \frac{\partial\langle V\rangle}{\partial \chi}=\left\langle(V-\langle V\rangle)^{2}\right\rangle .
$$

From this equation, substituting Eqs. (7) and (9), we obtain the following relation for the quantity $k$ :

$$
k=\frac{\partial\langle V\rangle}{\partial \chi}
$$

$k$ measures therefore the amount of volume that must be added to the system in order to increase of one "granular degree" the compactivity. The analogous quantity for molecular gases is denergy/dtemperature, which is the specific heat. In analogy with ordinary thermodynamics this "specific heat" is expected to be sensitive to changes in the system's internal properties.

\section{VORONOÏ VOLUME DISTRIBUTION}

We have tested the validity of the present theory over a set of several thousand numerical simulations and over 18 different experiments.
The numerical simulations consist of packings generated by means of a modified Lubachevsky-Stillinger algorithm [32-34]. The algorithm starts from random points in space and it makes them uniformly grow into nonoverlapping hard spheres with the sphere positions evolving in time according to Newtonian dynamics. The algorithm uses a cubic box and periodic boundary conditions. The simulation is ended when a jammed state with diverging collision rate is reached. Large expansion rates produce jammed configurations at low packing fractions whereas slower growth rates lead to larger packing fractions. For very slow rates, crystalline nuclei are formed. In our simulations we used 10000 spheres with unitary diameters obtaining jammed configurations with packing fractions between 0.56 and 0.65 by varying the growth rate between 500 and 0.000001 . We also generated nonjammed configurations in the range of packing fractions between 0.1 and 0.55 by keeping the growth rate at 0.001 and arresting the simulation once the desired packing fraction was reached. The independence of these unjammed configurations from the growth rate was also tested.

The experiments use both acrylic beads in air and glass beads in water obtaining different samples with packing fractions ranging from 0.56 to 0.64 . Details on these experimental systems are reported in $[27,29,30,41]$. Let us note that the fluidized bead experiments use a highly controlled technique which produces stationary packings with packing fractions which are independent of the preparation history and fluctuate around average values with smaller packing fractions for higher flow rates.

The fact that in all the above systems are at statistical equilibrium is a "working hypothesis" that appears to be supported by the very good agreement between the observations and the theoretical predictions (see later in this section).

Equation (6) is the main outcome of the present theory and it can be directly tested on the observations from experiments and computer simulations. The $k$-Gamma distribution predicted by Eq. (6) is valid for any aggregate of $k$ elementary cells. Therefore the same equation must describe both the volume fluctuations at the level of a single grain as well as the fluctuations at the level of the whole system.

\section{A. Local volume fluctuations}

Let us first study the system at the local level by investigating the volume fluctuations in the region around each grain. For this purpose we must first implement a method to assign a portion of volume associated to each grain in the packing. A natural way to divide space into cells "warped" around each grain is the Voronoï partition where cells are associated to the portion of space closest to a grain center with respect to any other center in the packing. Such a partition is particularly meaningful in the case of packing of equal spheres with unit diameter. In this case $V_{\min }=0.694$ [27] and the Voronoï cell is always circumscribing the internal sphere. Extension to grains with more generic shapes and sizes can be implemented.

Figure 1 shows the resulting distribution of the Voronoï volumes. One can see that such distributions span a very broad interval of volumes between 0.69 and 12 with large 

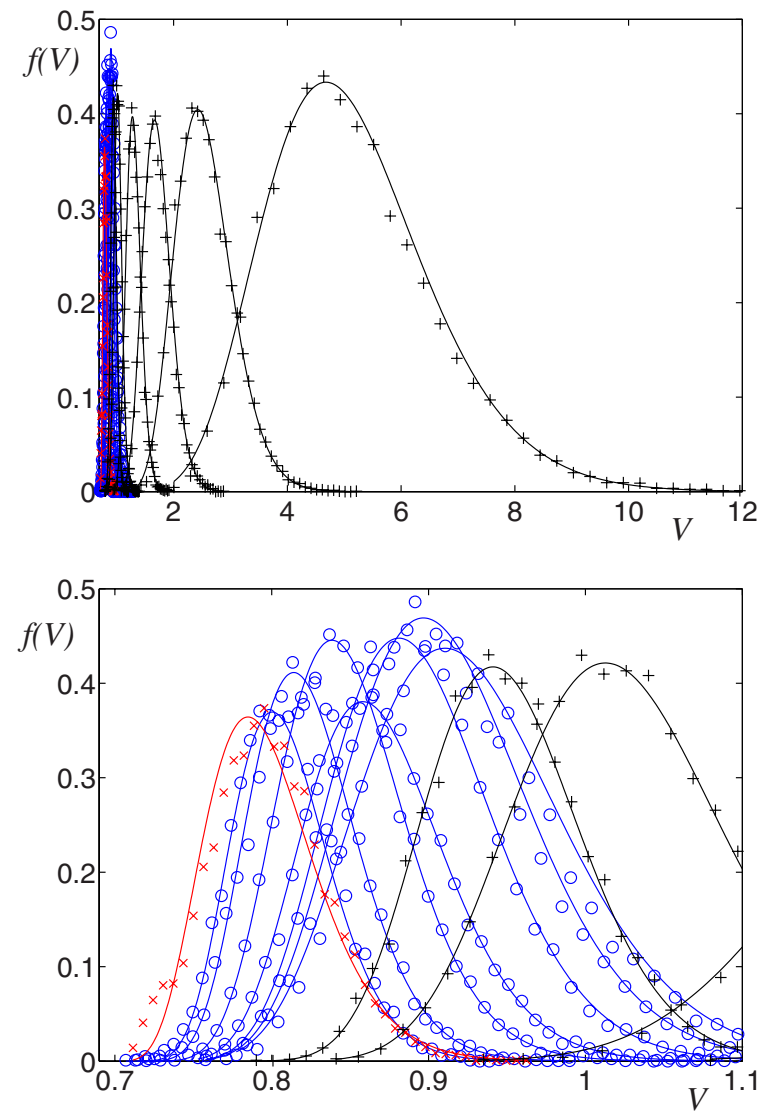

FIG. 1. (Color online) (Top) Voronoï volume distributions from numerically generated equal-sphere packings at packing fractions in a range between 0.1 and 0.65 . Different symbols refer to packings with different properties: jammed $(\bigcirc$ symbols), unjammed (+ symbols), and packing with crystalline inclusions ( $\times$ symbols). The lines are plots of Eq. (6) with the parameter $k$ calculated from $k$ $=\left(\bar{V}-V_{\min }\right)^{2} / \sigma_{v}^{2}$ [Eq. (7)]. (Bottom) The details of the jammed distributions at packing fractions between 0.55 and 0.65 .

differences between different samples. Lines in Fig. 1 are the plots of the $k$-Gamma distributions $f(V, k)$ [Eq. (6)] with the parameter $k$ calculated from $k=\left(\bar{V}-V_{\min }\right)^{2} / \sigma_{v}^{2}$ [Eq. (7)]. One can see that all the distributions follow well the theoretical prediction. However, given the broad and different domains in which such distributions are defined, a better visualization of such an agreement is necessary. This can be obtained by an appropriate shift and rescaling of the variable. Indeed, from Eq. (6), one can see that $k$-Gamma distributions characterized by similar values of $k$ must result in similar behaviors when plotted as a function of $\left(V-V_{\text {min }}\right) /\left(\bar{V}-V_{\text {min }}\right)$. Figure 2 shows the plot of all the distributions as a function of such a shifted-rescaled variable. We note that all the distributions from jammed configurations (bottom figure) collapse into very similar behaviors well described by $f(V, k)$ with $k$ in the range between 12.2 and 14.5. Unjammed configurations (top figure) also reveal very good agreement with $f(V, k)$ but in this case the parameter $k$ spans a larger interval $(9 \leq k \leq 25)$. We also note that the distribution with crystalline inclusions $(\times$ symbols $)$ deviates slightly from the predicted $k$-Gamma behavior revealing the emergence of a sec-
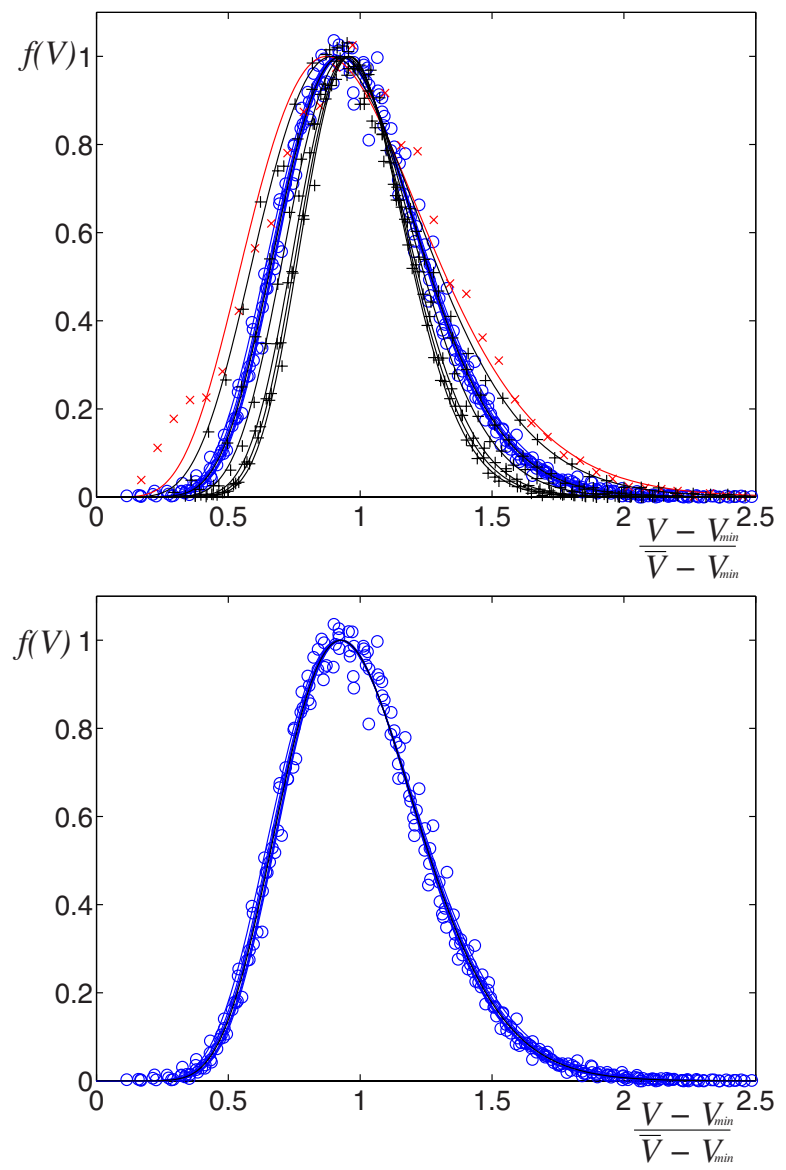

FIG. 2. (Color online) Voronoï volume distributions plotted vs $\left(V-V_{\min }\right) /\left(\bar{V}-V_{\min }\right)$. (Top) All distributions: jammed $(\bigcirc)$, unjammed $(+)$, and packing with crystalline inclusions $(X)$. (Bottom) The details of the jammed data show that they all collapse onto $k$-Gamma distributions in good agreement with Eq. (6) with very similar values of $k$ (between 12.2 and 14.5).

ond peak at small volumes (corresponding to the closepacked crystalline inclusions).

The numerical results analyzed in the previous paragraph refer to rather "ideal" packings made of nonoverlapping identical spheres. Remarkably, the same good agreement with a $k$-Gamma distribution $f(V, k)$ [Eq. (6)] was also found [27] in real experimental systems of acrylic spheres in air [27,29-31,42] and glass spheres in water [27] packed at different packing fractions by following different preparation protocols. Figure 3 shows that all the experimental distributions and the jammed configurations obtained numerically collapse into very similar behaviors well described by a $k$-Gamma distribution with $k$ in a narrow range around $k$ $\sim 12$. We note that the probability distribution function is well reproduced in all the range of volumes with a statistics performed over more than one million local configurations. This is verified over 18 different experimental systems and eight different computer simulations. Such systems span a range of packing fractions between 0.55 and 0.64 .

The impressive fact of such an agreement is that these systems are very different (ideal Newtonian spheres, acrylic beads in air, and glass beads in water) and they are prepared in very different ways (pouring, tapping, fluid flows, molecu- 




FIG. 3. (Color online) Comparison between the experimental Voronoï volume distributions for all 18 experimental samples with acrylic and glass beads in air and water [27] and all numerical jammed configurations $(\bigcirc)$ obtained by using the modified Lubachevsky-Stillinger algorithm [32-34]. Experiments with dry acrylic beads in air have symbols A $(\triangleleft), \mathrm{B}(\square), \mathrm{C}(\star), \mathrm{D}(\diamond), \mathrm{E}$ $(\triangle), F(\nabla)$. Experiments with glass beads in water have symbols + . When plotted vs $\left(V-V_{\min }\right) /\left(\bar{V}-V_{\min }\right)$ all the data collapse to a common behavior which is described well by a $k$-Gamma distribution with $k=12$ (black line).

lar dynamics, shearing). The collapse of all these distributions around $f(V, k \sim 12)$ suggests that there are universal properties that determine the packing configurations which are not very sensitive to the preparation method. Interestingly, we observe that unjammed configurations are also very well described by $k$-Gamma distributions but with parameters $k$ which are different from the one of the jammed case. Figure 4 reports the estimates for the parameter $k$ calculated from the relation $k=\left(\bar{V}-V_{\min }\right)^{2} / \sigma_{v}^{2}$ [Eq. (7)]. The value at zero packing fraction $(k=5.586)$ was calculated analytically for random Poisson points in three dimensions [39,43]. Figure 4 shows that, for nonjammed configurations, the value of $k$ increases almost linearly by increasing the packing fraction. Then the value of the parameter $k$ collapses around 12 when the system gets into a jammed configuration. The inset in the figure shows that there are sizable differences between different systems and between the same system at different packing fractions. A careful look shows a rather sharp variation in the value of $k$ for the experimental systems around the packing fraction $\rho \sim 0.6$. Special properties associated to the global volume fluctuations at $\rho \sim 0.6$ were also observed in fluidized bed experiments by Schröter et al. [20]. Above the packing fraction $\sim 0.645$, the simulations generate partially crystallized regions and the change in the kind of structural organization is revealed by a sharp drop in the value of $k$ that eventually will tend to zero in the crystalline limit. We observe that a $k$-Gamma distribution [Eq. (6)] with $k \sim 12$ is also followed by the Voronoï volumes from simulations of granular packings reported by Ciamarra et al. in Ref. [44]. An equivalent collapse for a completely different system concerning simulations of a polymer melt, water, and silica

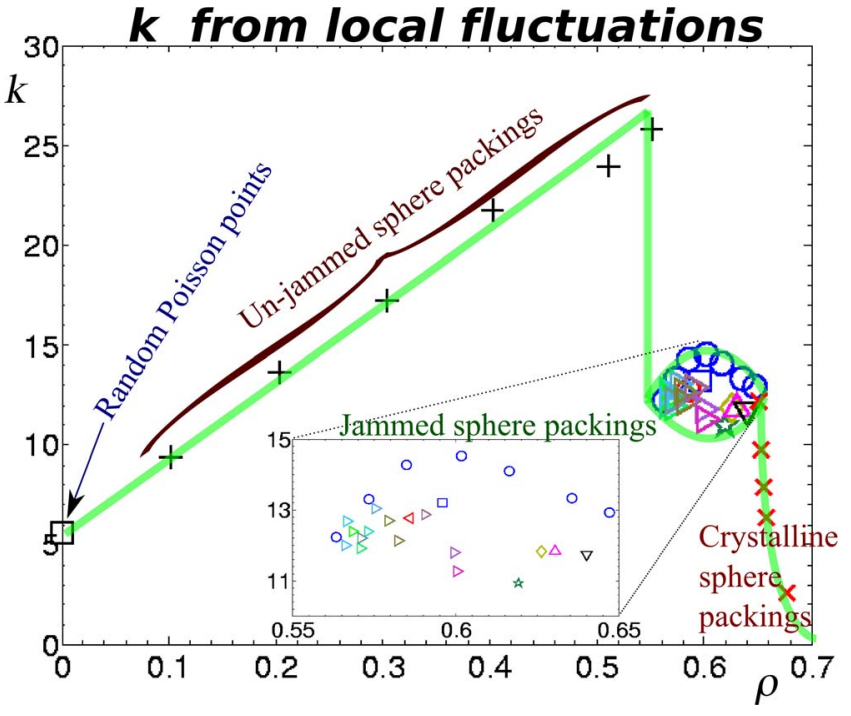

FIG. 4. (Color online) Behavior of $k$ calculated from $k=(\bar{V}$ $\left.-V_{\min }\right)^{2} / \sigma_{v}^{2}$ [Eq. (7)] vs packing fraction $\rho$. This plot refers to the statistics on local configurations computed over the fluctuations of the Voronoï cells' volumes. Sharp changes in $k$ are observed when spheres first jam $(\rho \sim 0.55)$ and when crystalline nuclei start to form $(\rho>0.65)$. The "+" refers to simulations of unjammed packings of spheres; the " $\bigcirc$ " are jammed packings simulated by using different growth rates and " $X$ " are jammed samples with crystalline inclusions; the symbols " $\square$ " correspond to 12 experiments with glass spheres in water prepared by means of the fluidized bed technique [27]; finally, the six symbols “ $\triangleleft, \square, \star, \diamond, \triangle, \nabla$ ” refer to six experiments with acrylic beads in air prepared by means of different methods [29-31,42].

was reported by Starr in Ref. [45] where volume distributions were plotted vs $(v-\langle v\rangle) / \sigma_{v}$. It is straightforward to see that such a scaling coincides with the one proposed in this paper, indeed $(v-\langle v\rangle) / \sigma=\sqrt{k}\left[\left(v-v_{\text {min }}\right) /\left(\langle v\rangle-v_{\text {min }}\right)-1\right]$. A careful look at the Starr data indicates a very good agreement with a $k$-Gamma distribution with $k \sim 20$.

\section{B. Global volume fluctuations of the whole system}

We have seen in the previous discussion that the volume fluctuations at the level of a single grain are very well described by $k$-Gamma distributions and we have observed that the value of the parameter $k$ is a good indication of the system's state (unjammed, jammed, polycrystalline). Let us now verify if the present theory can also correctly predict the volume fluctuations at the level of the whole sample. This requires the analysis of the distribution of the volumes of a large number of equivalent samples made by means of the same procedure. Differently from the single-grain case here we can only study jammed configurations. We have prepared such samples by using the modified Lubachevsky-Stillinger algorithm [32-34] generating a large number of jammed packings containing different numbers of grains (between $N=200$ and $N=10000)$ and prepared at different growth rates (between 0.0005 and 1000). For each growth rate and size we run at least 200 independent simulations and we measure the total volume occupied by the jammed spheres 




FIG. 5. (Color online) Fluctuations of the whole sample volume (symbols) from a large number of repeated simulations of jammed packings. The distributions are from samples containing $n=1000$ spheres each and generated with growth rates $0.01(\rho=0.637), 0.05$ $(\rho=0.616)$, and $1000(\rho=0.564)$. The lines are the $k$-Gamma distributions predicted theoretically from Eq. (6). They use no adjustable parameters: the parameter $k$ is calculated from $k=\left(\bar{V}-V_{\min }\right)^{2} / \sigma_{v}^{2}$ [Eq. (7)] and $V_{\min }=N / \sqrt{2}$.

$[V=\pi /(6 \rho)]$. Three examples of the resulting statistical distributions of volumes are shown in Fig. 5. Again we find that the $k$-Gamma distribution of Eq. (6) reproduces extremely well the observed fluctuations. The agreement is obtained without using any adjustable parameter. Indeed, to calculate the parameter $k$ we compute the average volume $\langle V\rangle=\bar{V}$ and the variance $\sigma_{v}^{2}=\left\langle V^{2}\right\rangle-\langle V\rangle^{2}$, and we use $k=\left(\bar{V}-V_{\min }\right)^{2} / \sigma_{v}^{2}$ [Eq. (7)]. In this case, $V_{\min }$ is fixed to the minimum volume attainable by a packing of $N$ equal spheres with unit diameter, which is the volume occupied by a fcc crystalline configuration, i.e., $V_{\min }=N / \sqrt{2}$.

One can note that the distribution tends to narrowing during compaction. The quantity $k$ is changing with the packing fraction and, for example, in the three examples in Fig. 5 we measure: $k=2.6 \mathrm{~N}$ at $\rho=0.564, k=4.4 \mathrm{~N}$ at $\rho=0.616$, and $k$ $=8.8 \mathrm{~N}$ at $\rho=0.637$. More generally, we compute the average volume and the variance for a very large set of data over a range of packing fractions within 0.55 and 0.66 . The resulting values of the parameter $k$ rescaled by the number of spheres in each sample $N$ are plotted in Fig. 6. We note that the data from samples with various sizes (between $N=200$ and $N=10000)$ all fall on similar values when rescaled by $N$ indicating that the parameter $k$ scales linearly with the number of spheres for large $N$ 's. The rescaled parameter $k / N$ takes values a little below 3 at the loose packing limit $(\rho$ $\sim 0.55)$. Then the value of the parameter grows consistently when the packing densifies and it reaches a peak at the random close packing limit $\rho \sim 0.64$. Afterwards, $k / N$ drops sharply and eventually goes to zero when the packing becomes crystalline. The small samples with 200 spheres show a slightly different behavior with the peak occurring before $\rho \sim 0.64$. We verified that this is a finite-size effect due to the formation of some polycrystalline samples.

It is therefore clear that the quantity $k$ is very sensitive to the changes in the system's internal structure and the peak at

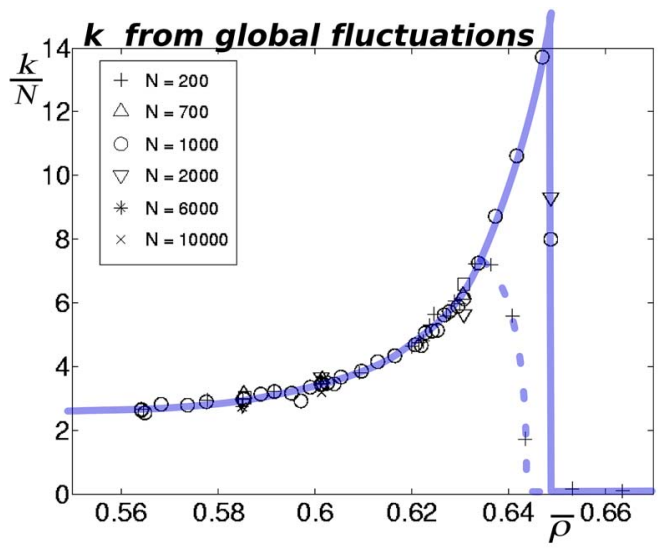

FIG. 6. (Color online) The parameter $k$ divided by the number of spheres in the sample $(N)$ is plotted vs the average packing fraction $\bar{\rho}=\pi /(6 \bar{V})$ for several samples with total number of spheres between 200 and 10000 . The value of $k$ is computed from $k / N$ $=1 / N\left(\bar{V}-V_{\min }\right)^{2} / \sigma_{v}^{2}=N\left(\frac{\pi}{6 \bar{\rho}}-\frac{1}{\sqrt{2}}\right)^{2} / \sigma_{v}^{2}$ over a large set of samples prepared by repeating a large number of simulations using the same growth rate for each average packing fraction. The full line is a guide for the eye showing the sharp transition occurring around 0.645 for samples with $N \geq 700$, and the dotted line highlights that small samples $(N=200)$ start the decay at smaller packing fractions around $\rho \simeq 0.635$.

the random close packing limit indicates that the packing must reorganize in order to proceed with compaction. A similar discontinuity at both the loose and close packing limits was observed in the study of $k$ from the local Voronoï volume statistics (Fig. 4). However, the values of the local quantity $(k)$ and the rescaled global quantity $(k / N)$ are different. This should not be of any surprise. Indeed, $k$ counts the number of elementary cells contributing to the system's volume. But, some elementary cells are sheared among neighboring Voronoï cells, and therefore the number that contributes to the volume of $N$ Voronoï cells is not necessarily $N$ times the number that contributes to one single cell. Furthermore, it is known that volume correlations play an important and unusual role in these systems [46]. It has been shown both in computer simulations and experiments [46-48] that the scaling of the volume fluctuations is not a simple linear function of the portion of sample investigated. Although not surprising, such different values and behaviors of the local and global $k$ have important implications. For instance, this implies that the granular temperature measured at the local level is different from the one measured from global fluctuations. In this respect, the "proper" granular temperature is the one associated to the global volume fluctuations, which we have shown is an intensive quantity since $k$ is scaling linearly with $N$ (at least for samples with more than 200 spheres, Fig. $6)$. The "temperature" associated with the local measure of $k$ is an "effective granular temperature." The fact that they predict so accurately the volume fluctuations both at the local and at the global level indicates that the effect of correlations and local geometrical constraints can be taken into account by simply tuning the value of $k$. 


\section{CONCLUSIONS}

In this paper we have obtained a $k$-Gamma distribution for the volume fluctuations by using a statistical mechanics approach " $a$ la Edwards" by introducing the following three assumptions: (i) the microscopic state can be encoded in terms of the properties of a set of $k$ elementary cells with volumes $v_{i}$; (ii) the cells' properties are either fully described by their volumes $v_{i}$ or they are independent from $v_{i}$; (iii) any space partition with cells of volumes $v_{i} \geq v_{\min }$ satisfying the condition $\sum_{i} v_{i}=V$ corresponds to an attainable packing. A comparison with a large number of experiments and computer simulations shows remarkably good agreement with the theoretical prediction in a wide range of packing fractions and for several differently prepared systems. Such an agreement holds both at the level of a single grain (Voronoï cells) and at the level of the whole system.

We have identified a quantity $k$ which is very sensitive to the system's structural organization. It results that such a quantity is the equivalent of the specific heat in ordinary thermodynamics. We have demonstrated that $k$ can be easily computed empirically [Eq. (7)] by measuring the average volume and the variance. A large number of measures from both the local statistics at the level of the single grain and the global statistics at the level of the whole sample show the signatures of clear structural transitions marked by peaks and discontinuities in the values of $k$ occurring at both the random loose packing and at the random close packing limits (Figs. 4 and 6). Similar peaks in the specific heat are observed at phase transitions in thermodynamic systems. Understanding whether proper thermodynamical phase transitions are occurring at the random loose packing limit and the random close packing limit is the subject of current investigations.

The remarkable quantitative agreement between theory, experiments, and simulations at both local and global levels is very encouraging, demonstrating that statistical mechanics is a powerful tool to study and predict the properties of com- plex materials. We have discussed that the local effects of correlations and geometrical constraints can be taken into account by using an "effective" local granular temperature which corresponds to a nonlinear scaling of $k$ with the number of grains. Further work must be devoted to better understand the link between local and global properties and identify the length scale above which the system becomes extensive. An important test to establish the correct granular temperature might use an independent measure from the fluctuation-dissipation theorem as discussed in [7,9]. The use of nonextensive forms of entropy instead of the classical Gibb's entropy might be the right approach to formulate a theory which consistently describes both local and global levels in terms of a unified generalized granular temperature.

Let us finally note that the agreement between theory and empirical observations is even excessively good. Indeed, some of the systems, such as the fluidized bed experiments, are likely to be at statistical equilibrium and they should be correctly described by equilibrium predictions. However, other systems, such as samples A and B, are more likely to be out of equilibrium, and therefore there are no a priori reasons why they should follow the same kind of statistical distribution. Furthermore, the same $k$-Gamma distribution has been observed in simulations of out of equilibrium colloid systems [49]. At the present we are investigating a different statistical mechanics approach based on Markov processes which might correctly describe the occurrence of $k$-Gamma distributions also in out of equilibrium systems [50].

\section{ACKNOWLEDGMENTS}

We thank Antonio Coniglio, Mario Nicodemi, Massimo Pica Ciamarra, Matthias Schröter, and Harry Swinney for helpful discussions. Many thanks to T. J. Senden, M. Saadatfar, A. Sakellariou, A. Sheppard, and A. Limaye for help with the tomographic data. This work was partially supported by the ARC discovery project DP0450292.
[1] P. Umbanhowar, Nature (London) 424, 886 (2003).

[2] M. Buchanan, Nature (London) 425, 556 (2003).

[3] M. van Hecke, Nature (London) 435, 1041 (2005).

[4] S. Edwards and R. Oakeshott, Physica A 157, 1080 (1989).

[5] S. Edwards, Granular Matter: An Interdisciplinary Approach (Springer-Verlag, New York, 1994), pp. 121-140.

[6] S. F. Edwards and D. V. Grinev, Phys. Rev. Lett. 82, 5397 (1999).

[7] A. Barrat, J. Kurchan, V. Loreto, and M. Sellitto, Phys. Rev. Lett. 85, 5034 (2000).

[8] A. Fierro, M. Nicodemi, and A. Coniglio, Europhys. Lett. 59, 642 (2002).

[9] H. A. Makse and J. Kurchan, Nature (London) 415, 614 (2002)

[10] B. Behringer, Nature (London) 415, 594 (2002).

[11] G. D’Anna, P. Mayor, A. Barrat, V. Loreto, and F. Nori, Nature (London) 424, 909 (2003).
[12] Y. Srebro and D. Levine, Phys. Rev. E 68, 061301 (2003).

[13] R. Blumenfeld and S. F. Edwards, Phys. Rev. Lett. 90, 114303 (2003).

[14] R. P. Ojha, P. A. Lemieux, P. K. Dixon, A. J. Liu, and D. J. Durian, Nature (London) 427, 521 (2004).

[15] J. H. Snoeijer, T. J. H. Vlugt, M. van Hecke, and W. van Saarloos, Phys. Rev. Lett. 92, 054302 (2004).

[16] P. T. Metzger, Phys. Rev. E 70, 051303 (2004).

[17] S. F. Edwards, J. Brujić, and H. A. Maskse, in Unifying Concepts in Granular Media and Glasses, edited by A. Coniglio, A. Fierro, and M. Nicodemi (Elsevier, Amsterdam, 2004), pp. 9-23.

[18] S. F. Edwards, J. Stat. Phys. 116, 29 (2004).

[19] P. Richard, M. Nicodemi, R. Delannay, P. Ribiere, and D. Bideau, Nat. Mater. 4, 121 (2005).

[20] M. Schröter, D. I. Goldman, and H. L. Swinney, Phys. Rev. E 71, 030301(R) (2005). 
[21] R. Blumenfeld and S. Edwards, Eur. Phys. J. E 19, 23 (2006).

[22] M. Pica Ciamarra, A. Coniglio, and M. Nicodemi, Phys. Rev. Lett. 97, 158001 (2006).

[23] F. Lechenault, F. da Cruz, O. Dauchot, and E. Bertin, J. Stat. Mech.: Theory Exp. 2006, 1742.

[24] P. T. Metzger and C. M. Donahue, Phys. Rev. Lett. 94, 148001 (2005).

[25] G.-J. Gao, J. Bławzdziewicz, and C. S. O'Hern, Phys. Rev. E 74, 061304 (2006).

[26] S. Henkes, C. S. O'Hern, and B. Chakraborty, Phys. Rev. Lett. 99, 038002 (2007).

[27] T. Aste, T. D. Matteo, M. Saadatfar, T. Senden, M. Schröter, and H. L. Swinney, Europhys. Lett. 79, 24003 (2007).

[28] M. Jerkins, M. Schröter, H. L. Swinney, T. J. Senden, M. Saadatfar, and T. Aste, e-print arXiv:0710.5160.

[29] T. Aste, M. Saadatfar, and T. J. Senden, Phys. Rev. E 71, 061302 (2005).

[30] T. Aste, M. Saadatfar, A. Sakellariou, and T. Senden, Physica A 339, 16 (2004).

[31] T. Aste, J. Phys.: Condens. Matter 17, S2361 (2005).

[32] B. D. Lubachevsky and F. H. Stillinger, J. Stat. Phys. 60, 561 (1990).

[33] A. Donev, S. Torquato, and F. H. Stillinger, J. Comput. Phys. 202, 737 (2005).

[34] M. Skoge, A. Donev, F. H. Stillinger, and S. Torquato, Phys. Rev. E 74, 041127 (2006).
[35] R. V. Hogg and A. T. Craig, Introduction to Mathematical Statistics (Macmillan, New York, 1978).

[36] A. Mehta and S. F. Edwards, Physica A 157, 1091 (1989).

[37] J. Brujić, S. F. Edwards, I. Hopkingson, and H. A. Makse, Physica A 327, 201 (2003).

[38] D. Weaire, J. P. Kermode, and J. Weichert, Philos. Mag. B 53, L101 (1986).

[39] E. Pineda, P. Bruna, and D. Crespo, Phys. Rev. E 70, 066119 (2004).

[40] E. Sultan and A. Boudaoud, Phys. Rev. Lett. 96, 136103 (2006).

[41] Additional material: http://wwwrsphysse.anu.edu.au/ granularmatter/.

[42] T. Aste, Phys. Rev. Lett. 96, 018002 (2006).

[43] E. N. Gilbert, Ann. Math. Stat. 33, 958 (1962).

[44] M. Pica Ciamarra, M. Nicodemi, and A. Coniglio, Phys. Rev. E 75, 021303 (2007).

[45] F. W. Starr, S. Sastry, J. F. Douglas, and S. C. Glotzer, Phys. Rev. Lett. 89, 125501 (2002).

[46] T. Aste and T. D. Matteo, Eur. Phys. J. E 22, 235 (2007).

[47] S. Torquato and F. H. Stillinger, Phys. Rev. E 68, 041113 (2003).

[48] A. Donev, F. H. Stillinger, and S. Torquato, Phys. Rev. Lett. 95, 090604 (2005).

[49] I. Schenker and L. J. Gauckler, (private communication).

[50] T. Aste and T. D. Matteo, e-print arXiv:0711.3239. 